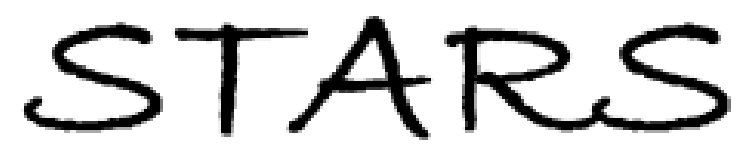

University of Central Florida

STARS

$1-1-2011$

\title{
A Novel Three-Protein Complex Regulates In Vitro Vtv-Golgi Fusion
}

\author{
S. A. Siddiqi \\ University of Central Florida \\ E. Nafi-Valencia \\ University of Central Florida \\ S. Siddiqi \\ University of Central Florida
}

A. Rahim

University of Central Florida

Find similar works at: https://stars.library.ucf.edu/facultybib2010

University of Central Florida Libraries http://library.ucf.edu

This Meeting Abstract is brought to you for free and open access by the Faculty Bibliography at STARS. It has been accepted for inclusion in Faculty Bibliography 2010 s by an authorized administrator of STARS. For more information, please contact STARS@ucf.edu.

\section{Recommended Citation}

Siddiqi, S. A.; Nafi-Valencia, E.; Siddiqi, S.; and Rahim, A., "A Novel Three-Protein Complex Regulates In Vitro Vtv-Golgi Fusion" (2011). Faculty Bibliography 2010s. 1920.

https://stars.library.ucf.edu/facultybib2010/1920

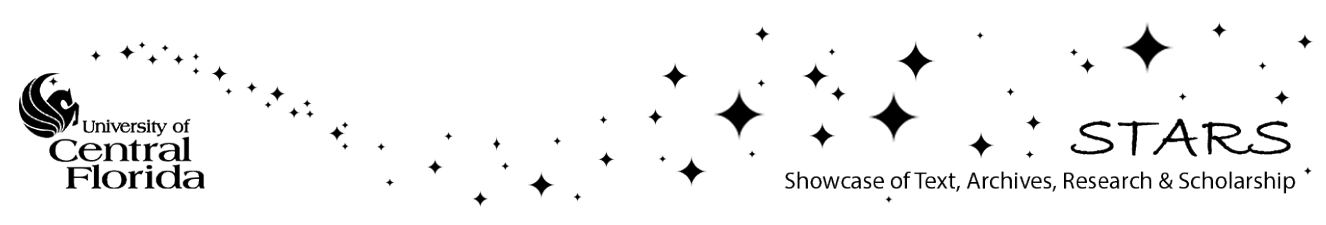


Conclusion: apoC1 is a constitutive inhibitor of CETP in normolipidemic humans, but it no longer operates in hyperlipidemic patients with CAD.

\section{ARAP2-INDUCED CHANGES IN SPHINGOLIPID BIOSYNTHESIS PROMOTE LIPID DROPLET FORMATION BY INCREASING GLUT1 LEVELS IN THE PLASMA MEMBRANE}

L. Li ${ }^{1}$, R. Mobini ${ }^{1}$, E. Lu ${ }^{1}$, M. Rutberg ${ }^{1}$, M. Ståhlman ${ }^{1}$, L. Håversen ${ }^{1}$, B. Liu ${ }^{1}$, T. Larsson ${ }^{1}$, R. Perkins ${ }^{1}$, L. Andersson ${ }^{1}$, K. Koistinen ${ }^{2}$, K. Ekroos ${ }^{2}$, J. Borén ${ }^{1}$, S.-O. Olofsson ${ }^{1}$. 1 Sahlgrenska Center for Cardiovascular and Metabolic Research, Wallenberg Laboratory, University of Gothenburg, Gothenburg, Sweden, ${ }^{2}$ Zora Biosciences Oy, Espoo, Finland

Lipid droplets are organelles involved in the storage and processing of neutral lipids such as triglycerides, and recent evidence suggests a close interaction between lipid droplets and specialized domains of the plasma membrane known as lipid rafts.

Here we identified ARAP2 as a lipid droplet-associated protein that increases the formation of lipid droplets by promoting triglyceride biosynthesis. ARAP2 increased basal glucose uptake and GLUT1 levels in the lipid raft fraction of the plasma membrane, which likely explains the increased triglyceride biosynthesis. We also showed that ARAP2 influenced the lipid composition of cells and the lipid raft fraction by promoting sphingomyelin biosynthesis and reducing glucosylceramide biosynthesis. Furthermore, we showed that ARAP2 could mediate these effects by inhibiting the activity of glucosylceramide synthase. Inhibition of this enzyme promoted the activity of plasma membrane sphingomyelin synthase 2 and the accumulation of GLUT1 in the lipid raft fraction of the plasma membrane. We also showed that palmitic acid increased the expression of ARAP2 and GLUT1 levels in the lipid raft fraction.

Our data indicate that GLUT1 levels in lipid rafts, and hence basal glucose uptake and triglyceride biosynthesis, can be increased by an ARAP2-induced reduction in GCS activity and in the glucosylceramide biosynthesis. We therefore propose that ARAP2 is part of a mechanism that protects cells from lipotoxic fatty acids by increasing basal glucose uptake to promote lipid droplet formation.

\section{A NOVEL THREE-PROTEIN COMPLEX REGULATES IN VITRO VTV-GOLGI FUSION \\ S.A. Siddiqi, E. Nafi-Valencia, S. Siddiqi, A. Rahim. Burnett School of} Biomedical Sciences, University of Central Florida, Orlando, FL, USA

Elevated concentrations of circulating plasma very low-density lipoproteins (VLDLs) play an important role in the pathogenesis of atherosclerosis. VLDLs are synthesized in the liver and secreted into the blood. The rate-determining step in the secretion of VLDLs from the liver is their transport from their site of biogenesis, the endoplasmic reticulum (ER), to the Golgi. We reported recently that this physiologically regulatable step is mediated by a specialized ER-derived VLDL transport vesicle (VTV). VTV fuses with hepatic cis-Golgi to facilitate the targeted delivery of VLDL to the Golgi lumen. In vitro fusion of VTV with hepatic cis-Golgi requires cytosol, however, cytosolic factors regulating fusion event remain unidentified. To identify cytosolic factors, we performed a series of chromatographic steps including FPLC on liver cytosol and isolated a complex of three cytosolic proteins, which we found is required for in vitro VTV-Golgi fusion. Tandem mass spectrometry coupled with N-terminal sequencing analyses identified the proteins as hsp72, p97 and hsp27. Western blotting data using antibodies to hsc70, p97 and hsp27 confirmed their identity. To examine the functionality of these proteins in VTV-Golgi fusion, we immunodepleted hepatic cytosol of hsc70, p97 and hsp27 using specific antibodies. Immunodepletion of each of the three proteins resulted in significant reduction in VTV-Golgi fusion. Addition of recombinant hsc70, p97 and hsp27 proteins to the immunodepleted-cytosol completely restored the fusion activity. We conclude that hsc70, p97 and hsp27 form a complex in cytosol, which regulates the fusion VTV with hepatic cis-Golgi and thus VLDL-delivery to the Golgi lumen.

\section{MURINE BONE MARROW-DERIVED MACROPHAGES DIFFERENTIATED WITH GM-CSF BECOME FOAM CELLS BY PI 3-KINASE GAMMA-DEPENDENT FLUID-PHASE PINOCYTOSIS OF NATIVE LDL}

J.J. Anzinger ${ }^{1}$, J. Chang ${ }^{1}$, Q. Xu ${ }^{1}$, T. Bohnacker ${ }^{2}$, M.P. Wymann ${ }^{2}$, H.S. Kruth ${ }^{1}$. ${ }^{1}$ Experimental Atherosclerosis Section, NHLBI, NIH, Bethesda, MD, USA, ${ }^{2}$ Department of Biomedicine, Institute of Biochemistry and Genetics, University of Basel, Basel, Switzerland

Accumulation of cholesterol by macrophage LDL uptake is a key event in the formation of atherosclerotic plaques. Previous research has shown that GMCSF differentiates macrophages, is present in atherosclerotic plaques, and promotes plaque lipid accumulation. However, it has not been determined if murine GM-CSF-differentiated macrophages take up LDL to become cholesterol-rich foam cells. In this study, murine bone marrow-derived cells were differentiated into macrophages with GM-CSF. These GM-CSF differentiated macrophages showed massive, progressive accumulation of cholesterol during incubation with LDL. Incubation of LDL receptor-null or wild-type macrophages with increasing concentrations of ${ }^{125}$ I-LDL showed non-saturable macrophage LDL uptake that was linearly related to the LDL amount added, indicating receptor-independent LDL uptake mediated by fluid-phase pinocytosis. Since previous studies showed an important role for PI3K in atherosclerosis development and macrophage fluid-phase pinocytosis, we investigated the role of PI3K in mediating macrophage fluid-phase pinocytosis of LDL. Wild-type macrophages incubated with LDL and the PI3K-gamma inhibitor AS605240 showed a $\sim 50 \%$ reduction in LDL uptake and net cholesterol accumulation compared with macrophages incubated with LDL only. Incubation of PI3Kgamma-null macrophages with LDL showed a similar $\sim 50 \%$ reduction in LDL uptake and net cholesterol accumulation compared with wild-type macrophages incubated only with LDL. These results show that GM-CSF-differentiated murine macrophages become cholesterol-rich foam cells by fluid-phase pinocytosis of native LDL and identify PI3K-gamma as contributing to this process. PI3K-gamma-dependent macrophage foam cell formation mediated by fluidphase pinocytosis provides mechanistic insight for the decreased vascular lipid accumulation previously observed during atherosclerosis development in mice lacking PI3K-gamma.

\section{LIPID DROPLETS: STRUCTURE, PROTEIN ORGANIZATION AND BIOGENESIS}

H. Robenek ${ }^{1}$, N.J. Severs ${ }^{2}$, G. Weissen-Plenz ${ }^{1}$, A. Ruebel ${ }^{1}$, I. Buers ${ }^{1} .{ }^{1}$ Cell Biology and Ultrastructure Research, Leibniz-Institute for Arteriosclerosis Research, Univ. Muenster, Münster, Germany, ${ }^{2}$ Heart and Lung Institute, Imperial College London, London, UK

An entirely new view of lipid droplets is emerging: lipid droplets are not merely storage depots for superfluous intracellular lipids in times of hyperlipidic stress, but metabolically active organelles involved in cellular homeostasis. Virtually all recent advances in our concepts on the metabolic functions of lipid droplets have come from studies on lipid droplet-associated proteins. This realization has made the study of proteins, such as PAT family proteins, caveolins and several others that are targeted to lipid droplets, an intriguing and rapidly developing area of intensive inquiry. Despite the progress in lipid droplet research, however, the lipid droplet is still a poorly understood organelle. Our existing understanding of the structure, protein organization and biogenesis of the lipid droplet has relied heavily on microscopical techniques that lack resolution and the ability to preserve native cellular and protein composition. The electron microscopic technique, freeze-fracture replica immunogold labeling, overcomes these disadvantages and can be used to define at high resolution the precise location of lipid droplet-associated proteins in specific membrane systems and organelles of the cell. In this presentation illustrative examples of how freeze-fracture immunocytochemistry has contributed to our understanding of the identification, spatial organization in the membrane plane as well as the dynamic cellular processes and function of PAT family proteins and caveolin-1 are shown. By revisiting the lipid droplet and its associated proteins with freeze-fracture immunocytochemistry, new perspectives have emerged which challenge prevailing concepts of lipid droplet biology and may hopefully provide a timely impulse for many ongoing studies.

\section{TRIACYLGLYCEROL-RICH LIPOPROTEINS INFLUENCE ON THE INACTIVATION OF LIPOPROTEIN LIPASE BY ANGPTL 3 AND ANGPTL 4}

S. Nilsson ${ }^{1}$, M. Larsson ${ }^{1}$, E. Worrsjö ${ }^{1}$, A. Lookene ${ }^{1,2}$, V. Suokonina ${ }^{1,3}$, E. Makoveichuk ${ }^{1}$, J. Heeren ${ }^{4}$, G. Olivecrona ${ }^{1}$. ${ }^{1}$ Physiological Chemistry, Umeå University, Umeå, Sweden, ${ }^{2}$ Tallinn University of Technology, Tallinn, Estonia, ${ }^{3}$ Biomedicine, University of Gothenburg, Göteborg, Sweden, ${ }^{4}$ IBMII: Molecular Cell Biology, University Medical Center Hamburg-Eppendorf, Hamburg, Germany

Objectives: Angiopoietin-like proteins (ANGPTL3 and -4) are present both in tissues and in blood and have been found to modulate the activity of LPL. We wanted to investigate if lipoproteins influence on this interaction and if ANGPTL3 and -4 could influence LPL-dependent, receptor-mediated endocytosis of lipoproteins by primary hepatocytes.

Methods: LPL activity was measured after pre-incubation in the presence of ANGPTL3 or -4 with or without chylomicrons, VLDL or LDL. Binding of ANGPTL3 and -4 to the lipoproteins was investigated by SPR and ELISA. LPL-dependent uptake of ${ }^{125}$ I-labeled triacylglycerol-rich lipoproteins (TRLs) in hepatocytes was investigated in the presence or absence of ANGPTL3 and -4 . Results: TRLs significantly reduced the inactivation of LPL by ANGPTL3 and -4 , while LDL had less effect on the inactivation. LPL bound to the TRLs, while ANGPTLs did not bind. The ANGPTLs were also found to decrease LPLdependent uptake of ${ }^{125}$ /-labeled TRLs in primary hepatocytes.

Conclusions: Our data show that LPL is protected from inactivation by ANGPTLs already at sub-physiological levels of TRLs, implying that circulating ANGPTLs may have little effect on LPL at the luminal side of the endothelium. This may explain why there is little correlation between plasma concentrations of ANGPTLs and plasma TG levels or LPL activity in tissues. Our data 